\section{P2-S7.03 PREGNANCY AND CONTRACEPTIVE EXPERIENCES OF WOMEN LIVING WITH HIV IN MEXICO}

doi:10.1136/sextrans-2011-050108.370

M van Dijk, K Wilson, X Contreras, A Ettenger, H D Fukuda, S García. Population Council Mexico Office Mexico D.F. Mexico

Background In Mexico new HIV infections are occurring increasingly in women, but little is known about the experiences of women with HIV/AIDS seeking sexual and reproductive healthcare.

Methods In four states we conducted in-depth interviews with HIVpositive women who were pregnant or had been pregnant within the last 5 years. Participants were recruited through hospitals and clinics providing HIV care and through NGOs providing health and social services for people living with HIV.

Results Participants reported that the information and care they received were inadequate and influenced more by their providers' personal opinions than by their own fertility desires. They experienced provider discrimination and pressure to use particular contraceptive methods. HIV specialists and OB/GYNS differed significantly in practice, with HIV specialists focusing on prevention and condom use while OB/GYNS focused on long-acting or permanent methods of contraception. Due to gaps in contraceptive counselling and options many women had unplanned pregnancies while others resorted to sterilisation or abstinence. Furthermore, few women received adequate prenatal HIV care and counselling on pregnancy options.

Conclusions The health sector seems to focus narrowly on disease prevention and treatment lacking a holistic approach to women's wellbeing. The experiences of women living with HIV/AIDS reveal discrepancies between policy and practice in Mexico as manifested in gaps and inconsistency in healthcare services and even failures to respect patient confidentiality and human rights. The health sector should improve the integration of HIV and sexual and reproductive healthcare through cross-training HIV specialists and OB/GYNS, and by continued sensitisation of all providers on the topic.

\section{P2-S7.04 MAXIMISING THE IMPACT OF POSITIVE PREVENTION PRACTICES IN PEOPLE LIVING WITH HIV: LEARNING'S FROM KARNATAKA, SOUTH INDIA}

doi:10.1136/sextrans-2011-050108.371

${ }^{1} \mathrm{~K}$ Jayanna, ${ }^{1} \mathrm{R}$ Washington, ${ }^{2} \mathrm{~S}$ Moses. ${ }^{1}$ Karnataka Health Promotion Trust, Bangalore, India; ${ }^{2}$ University of Manitoba, Winnipeg, Canada

Background We tried to understand the positive prevention practices in people living with HIV in Karnataka with specific focus on behaviours related to seeking treatment for sexually transmitted diseases and condom use. This gives an indication of programmatic efforts to further halt the transmission of virus and promote healthy behaviours within communities.

Methods During 2010, a cross-sectional survey was administered to 282 PLHIV across three districts of Karnataka in the context of large scale HIV care and support program. Information related to positive prevention practices surrounding STIs and condom use were collected, as well as socio-demographic details.

Results Of the eligible 338 PLHIV, 283 participated with a response rate of $83.7 \%$ (144 men and 139 women). Majority of the men were married $(66 \%)$ while women were either widowed or separated $(57 \%)$. Knowledge about STIs among women was better than men $(94.8 \%, 51.1 \%)$, but women reported more STIs in the last one year compared to men $(36.3 \%$ : $14.7 \%, \mathrm{p}<0.001) .17 .5 \%$ of never married, $21 \%$ of married and $33 \%$ of separated/ widowed also reported STIs. $98.6 \%$ of those who reported STIs had accessed treatment, but only $64.7 \%$ completed treatment for any particular STI. Treatment completion rate for separated or divorced PLHIVs was poorest (60.7\%). Reported condom use with regular partners was reported as lowest by the widowed persons (50\%) followed by elderly PLHIVs (61\%) and never married (71\%). People who were contacted by peer supported programs reported better knowledge about STIs and more consistent condom use with regular partners ( $86 \%$ vs $78 \%, \mathrm{p}<0.01)$. Conclusions The study reveals that certain profiles of people living with HIV such as females in general and widows in particular younger and never married PLHIVs are more vulnerable than the rest and hence need more attention in terms of prevention efforts by the programs. Better micro-planning and peer mediated outreach efforts focused on these profiles can yield better impact in resource constrained settings.

\section{P2-S7.05 ASSESSING THE QUALITY OF LIFE OF HIV/AIDS PATIENTS ON ARV THERAPY AND THEIR CAREGIVERS AT THE NATIONAL HOSPITAL ABUJA}

doi:10.1136/sextrans-2011-050108.372

${ }^{1} \mathrm{~K}$ Arogunyo, ${ }^{2} \mathrm{C}$ Asuzu. ${ }^{1}$ University of Ibadan, Ibadan, Nigeria; ${ }^{2}$ \& $R$ Communications

The study assessed the quality of life of HIV/AIDS patients on Antiretroviral (ARV) Therapy and their caregivers at the National Hospital, Abuja. A sample of 200 participants comprising 150 patients and 50 caregivers was select for the study using the simple random sampling technique. The sample was drawn from the population of HIV/AIDS patients receiving ARV therapy from the Special Treatment Clinic (STC) at the National Hospital, Abuja. The sample of the caregivers was as well drawn from the same source. Three scales were adapted for use to collect data. They include: World Health Organisation Quality of Life bref, Zung Depression Guide Rating Scale and Zung Self-Rating Anxiety Scale. The instruments have a reliability coefficients of $0.78 ; 0.69$, and 0.90 respectively. Also, a self-designed scale was used to measure the patients' drug adherence behaviour. Data generated were subjected to statistical manipulations using such tools as Analysis of Variance (ANOVA), t test, $\chi^{2}$, Pearson Product Moment Correlation and Multiple Regression Analysis. Also, scores of patients on the QOL scale were correlated with those of the caregivers. Seven hypotheses were tested for significance at 0.05 level and findings reveal that: though HIV/AIDS affects quality of life of AIDS patients, they are nonetheless able to live fulfilled lives and function normally when there is psychosocial support, enhanced social interaction and personal environmental health. Caregivers differ a great deal from patients in their quality of life, signifying the negative impact of the disease on the patients. Also revealed is that caregivers' quality of life does not significantly influence patients' quality of life ( $p$ value $>0.05$ ). Again, it was also discovered that patients' ARV therapy adherence behaviour does not significantly impact on quality of life ( $p$ value $>0.05$ ). Other factors were therefore suspected to be contributing more to $\mathrm{QOL}$ of patients than mere therapy adherence. Patients' social demographic characteristics jointly contribute to quality of life. And when isolated, patients' job status and their religious belief/affiliations impact significantly on QOL. The mean scores of patients on the four domains of QOL were compared. Patients' performance was highest in the environmental health domain, followed by that of social relationship domain and then, the psychological health domain. Physical health domain had the least mean score. Meanwhile, scores of the four domains of quality of life jointly impact significantly to the overall quality of life of patients. Further, scores on depression and anxiety were compared with those of QOL and result showed that both depression and anxiety reduce patients' quality of life with depression making the strongest negative impact on quality of life. However, gender, age and marital status do not make any significant difference in quality of life among patients. Implications of these findings were further discussed and effective measures for improving quality of life of patients of HIV/AIDS were recommended. 